\title{
A FIELD-CONSISTENT TWO-NODED CURVED AXISYMMETRIC SHELL ELEMENT
}

\author{
C. RAMESH BABU AND G. PRATHAP \\ Structural Sciences Division, National Aeronautical Laboratory, Bangalore 560017, India.
}

\section{SUMMARY}

The efficiency of a field-consistent two-noded linear curved axisymmetric shear-flexible shell element is shown to be due to the removal of both shear and membrane locking. Typical applications illustrate how the field-consistent representation dramatically improves performance, and allows greater flexibility in tailoring element design to satisfy specific problem needs.

\section{INTRODUCTION}

A very efficient two-noded straight element for application to axisymmetric shells was presented by Zienkiewicz et al.' This included shear deformation effects and yet was able to produce very successfully the Kirchhoff type of behaviour, even for very thin shells. This was made possible by the use of a single point numerical integration for the shear strain energy, thereby relieving the shear locking effect. Since the element was used as a linear segment, the problem of membrane locking was not encountered. A curved quadratic shear flexible element was attempted by Mohr, ${ }^{2}$ but again, apparently due to membrane locking, the contribution to meridional membrane strain was omitted. This cannot be without error in applications to curved shells of revolution. In this paper, therefore, we shall examine critically the use of a curved linear element and show how the shear and membrane locking phenomena operate and how they can be removed by employing field-consistent definitions for the constrained strain fields.

The field-consistency approach is a better procedure compared to the reduced integration technique, as it frees the restriction on the integration rules and allows the use of any uniform integration rule depending on the specific problem requirements. This aspect will be demonstrated in this paper through numerical examples.

\section{BACKGROUND TO FIELD-CONSISTENCY THEORY}

We recognize that a shear-flexible curved axisymmetric shell element is a problem in structural mechanics, which needs a description of its continuum behaviour by three independent field variables, the tangential displacement $\mathrm{u}$, the radial displacement $\mathrm{w}$ and the section rotation $\mathrm{fl}$, based on a general curvilinear system (Figure 1). The extensional, flexural and shear strains associated with this description can now be written as

$$
\{\mathrm{E}\}=\left\{\begin{array}{lllll}
\mathrm{Es} & \text { go } & \mathrm{Xs} & \mathrm{X}_{0} & \mathrm{Y}
\end{array}\right\}
$$



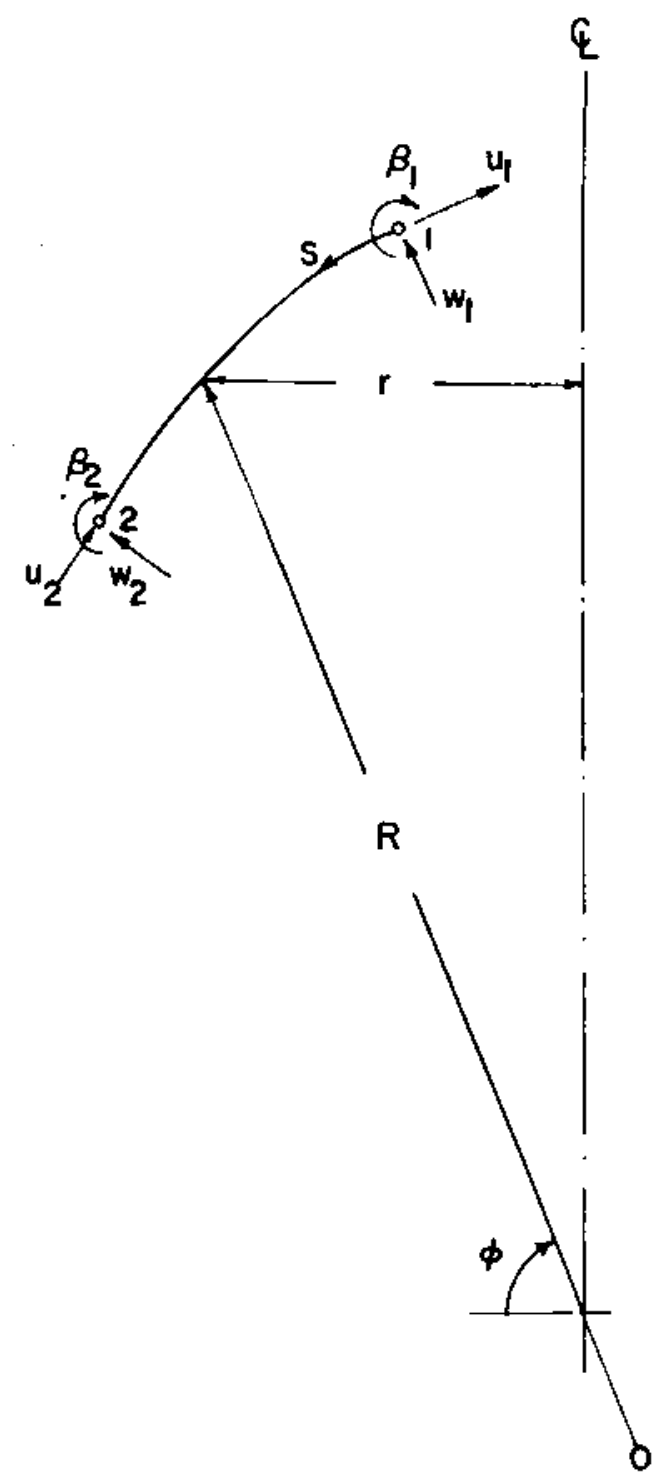

Figure 1. Curved axisymmetric linear shelt element

where

and

$$
\begin{aligned}
& \varepsilon_{s}=u_{. s}+w / R \\
& \varepsilon_{\theta}=(w \cos \phi+u \sin \phi) / r \\
& \chi_{s}=-\beta_{. s}+(u / R)_{, s} \\
& \chi_{\theta}=-\sin \phi(\beta-u / R) / r
\end{aligned}
$$

$$
\gamma=w_{, s}-\beta
$$

In many practical situations, the engineering dimensions of the problem require that certain of the strain fields that arise above have to be constrained in particular ways. Thus, in very thin shells, the Kirchhoff constraint emphasizes the vanishing of shear strains. In a doubly curved shell of revolution, an inextensional type of bending will require that the extensional strains must be able to vanish. However, with low order elements, the interpolation functions cannot produce 
strain fields which can establish only the true constraining conditions required in these cases. Failure to do so leads to the shear locking and membrane locking phenomenon. We shall call such representations field-inconsistent. We shall show below how the use of field-consistent interpolations for the strain fields can produce very efficient low order elements.

\section{Field-consistent shear strains}

Consider the definition of shear strain which arises out of the use of linear interpolations for $w$ and $\beta$, i.e.

$$
\begin{aligned}
& w=b_{0}+b_{1} s \\
& \beta=c_{0}+c_{1} s
\end{aligned}
$$

so that

$$
\gamma=w_{, s}-\beta=\left(b_{1}-c_{0}\right)-c_{1} s
$$

In the Kirchhoff limit, which we expect for thin shells, the requirement that shear strains will vanish produces, for the strain field described by equation (2) above, the following constraints:

$$
\begin{aligned}
b_{1}-c_{0} & =0 \\
c_{1} & =0
\end{aligned}
$$

Clearly, equation (3a) represents a constraint involving contributions from the field interpolations for $w$ and $\beta$ and can therefore reproduce a true constraint of the type $\left(w_{s}-\beta\right)_{0}=0$ where the subscript ' 0 ' denotes a reference point in the element. Equation (3b), however, represents a constant term only from the interpolation for $\beta$, and therefore behaves as a spurious constraint restricting the flexibility of the interpolation for $\beta$. This can be interpreted as an unnecessary constraint of the type $\beta_{s}=0$ within the element. A previous exercise ${ }^{3}$ has shown that this field-inconsistent term is the source of shear locking.

A simple solution to this problem is the use of a smoothed, field-consistent interpolation for shear strain. In this case, this is simply

$$
\bar{\gamma}=b_{1}-c_{0}
$$

The same result can be achieved by the use of a single point integration for the shear strain energy. ${ }^{1}$ We feel that a clearer understanding of the mechanics involved here emerges if field-consistent strain interpolations are used in this manner and a uniform order of numerical integration is used for all energy terms. Again, there is now no ambiguity about where to sample the shear strains--the field-consistent interpolation is valid over the element. In addition, there is no longer a restriction on the order of integration as this can now be determined by other considerations, as we shall see in examples later.

\section{Field-consistent membrane strains}

A very similar mechanism operates to produce the membrane locking effect. ${ }^{4}$ The crucial term is now the meridional extensional strain $\varepsilon_{s}$. An application of the same logic used above will suggest that where shape functions of the form

$$
\begin{aligned}
& u=a_{0}+a_{1} s \\
& w=b_{0}+b_{1} s
\end{aligned}
$$


are used, a field-consistent strain interpolation which will preclude membrane locking will be

$$
\bar{\varepsilon}_{s}=\left(a_{1}+b_{0} / R\right)
$$

Our investigations also indicate that improved accuracy is obtained for axisymmetric cylindrical shells, when $\varepsilon_{\theta}$ is also made field-consistent with $\varepsilon_{s}$. This is required, as in such problems we require $\left(\varepsilon_{s}+v \varepsilon_{\theta}\right)_{, s}=0$ and field-consistency is achieved at this level only if $\varepsilon_{\theta}$ is also a constant. Thus

$$
\varepsilon_{\theta}=\left(b_{0} \cos \phi+a_{0} \sin \phi\right) / r
$$

Formulation of the stiffness matrix

The appropriate elasticity matrices must represent the membrane, flexural and shear rigidities. Following Reference 1 , these are taken as

For isotropic material, these are

$$
\mathbf{D}=\left[\begin{array}{lll}
\mathbf{D}_{1} & & \\
& \mathbf{D}_{2} & \\
& & D_{3}
\end{array}\right]
$$

$$
\mathrm{D}_{1}=\frac{E t}{1-v^{2}}\left[\begin{array}{ll}
1 & v \\
v & 1
\end{array}\right], \quad \mathrm{D}_{2}=\frac{E t^{3}}{12\left(1-v^{2}\right)}\left[\begin{array}{ll}
1 & v \\
v & 1
\end{array}\right], \quad D_{3}=\frac{k E t}{2(1+v)}
$$

where $E$ is the Young's modulus, $v$ is the Poisson's ratio, $k=5 / 6$ is the shear correction factor and $t$ is the shell thickeness.

The strain energy of the system is now

$$
U=\frac{1}{2} \int_{0}^{s} \varepsilon^{\top} D \varepsilon 2 \pi r d s
$$

The strains are now interpolated in the field-consistent way described above and the strain energy terms are integrated exactly using a single integration order. In this case, the presence of linear interpolation for $\varepsilon_{\theta}$ and $\chi_{\theta}$ suggests that for mathematical completeness, a two point rule will be correct. However, Zienkiewicz has shown that a one-point rule would not lead to a large loss of accuracy. We shall adopt a two-point rule throughout for all energy terms in most cases, and higher order rules where accurate integration of the $1 / r$ terms that occur in the strain description provides greater accuracy in some cases.

\section{Improved geometric representation}

We see from equations (1) that the radius $r$ enters into the strain delinitions. In Reference 1, where each curved beam element is represented by a straight line segment and a one-point integration is used, the value $r_{\mathrm{m}}$ used at the integrating point is obtained as the average of the values of $r_{1}$ and $r_{2}$ at the nodes. In the present investigations, as each segment is replaced by a curved element of constant radius of curvature $R$, one can obtain an improved representation for $r(s)$. To do this, we start with the basic relations compatible with such a geometrical approximation, namely

and

$$
\phi_{, s}=1 / R
$$

$$
r_{, s}=-\sin \phi
$$


Integrating these, we obtain the simple representation

and

$$
r(s)=r_{1}+R\left\{\cos \phi(s)-\cos \phi_{1}\right\}
$$

$$
\phi(s)=\phi_{1}-s / R
$$

We shall use this definition to determine $r(s)$ at the integration points.

\section{NUMERICAL EXAMPLES}

The following test examples are chosen to demonstrate clearly the mechanisms operating to cause shear and membrane locking. Several element variations are necessary to compare these effects. The variations studied here are

(a) IMIS-the original field-inconsistent definitions for membrane and shear strains

(b) IMCS-field-inconsistent membrane and field-consistent shear strain

(c) CMIS-field-consistent membrane and field-inconsistent shear strain

(d) $\mathrm{CMCS}-$ field-consistent membrane and shear strains

Simply supported circular plate with uniformly distributed load

Figure 2 shows a circular plate, simply-supported along the circumference. The dimensions and material properties are $R=1 \mathrm{~m}, E=10^{6} \mathrm{kN} / \mathrm{m}^{2}, v=0$ and $P=1 \mathrm{kN} / \mathrm{m}^{2}$. The full plate is analysed with $5,10,20$ and 40 elements. As there is no membrane action here, numerical experiments were carried out using the IMIS and IMCS elements. The effect of shear locking can be well brought out by using the additional stiffness parameter as the error norm, where this error norm is

$$
e=W / W_{\text {FEM }}-1
$$

In this case, $W$ is the theoretically predicted deflection at the centre of the plate and $W_{\text {FEM }}$ is that computed from the finite element model. If shear locking exists in IMIS, $e$ should deteriorate in an $(L / t)^{2}$ fashion, where $L$ is the length of the element and $t$ is the plate thickness. Figure 3 shows, on a logarithmic plot, that IMIS locks exactly in this fashion. Errors from the IMCS model were too small to be shown on this graph and were virtually unchanged as $L / t$ is increased,

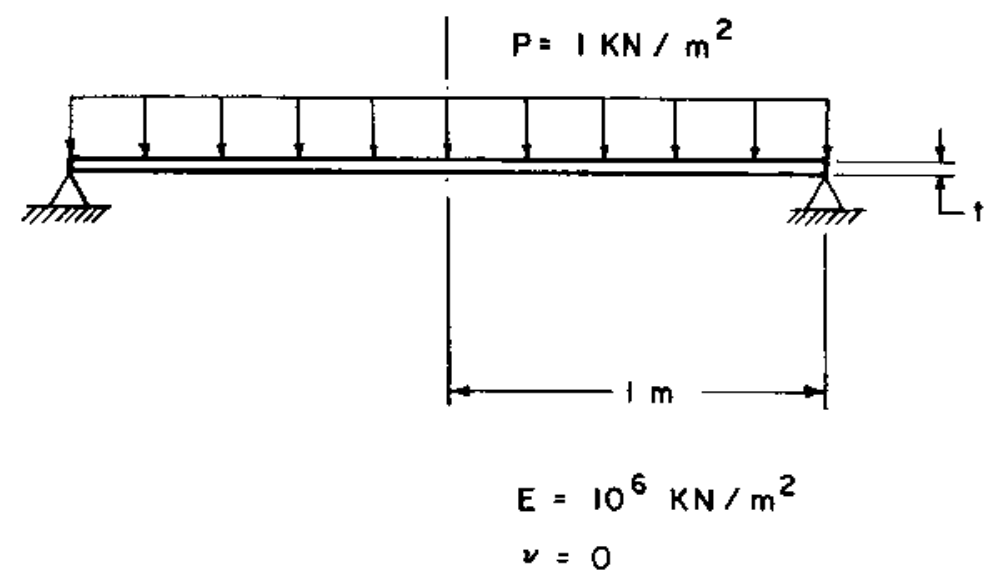

Figure 2. Simply supported circular plate under UDL 


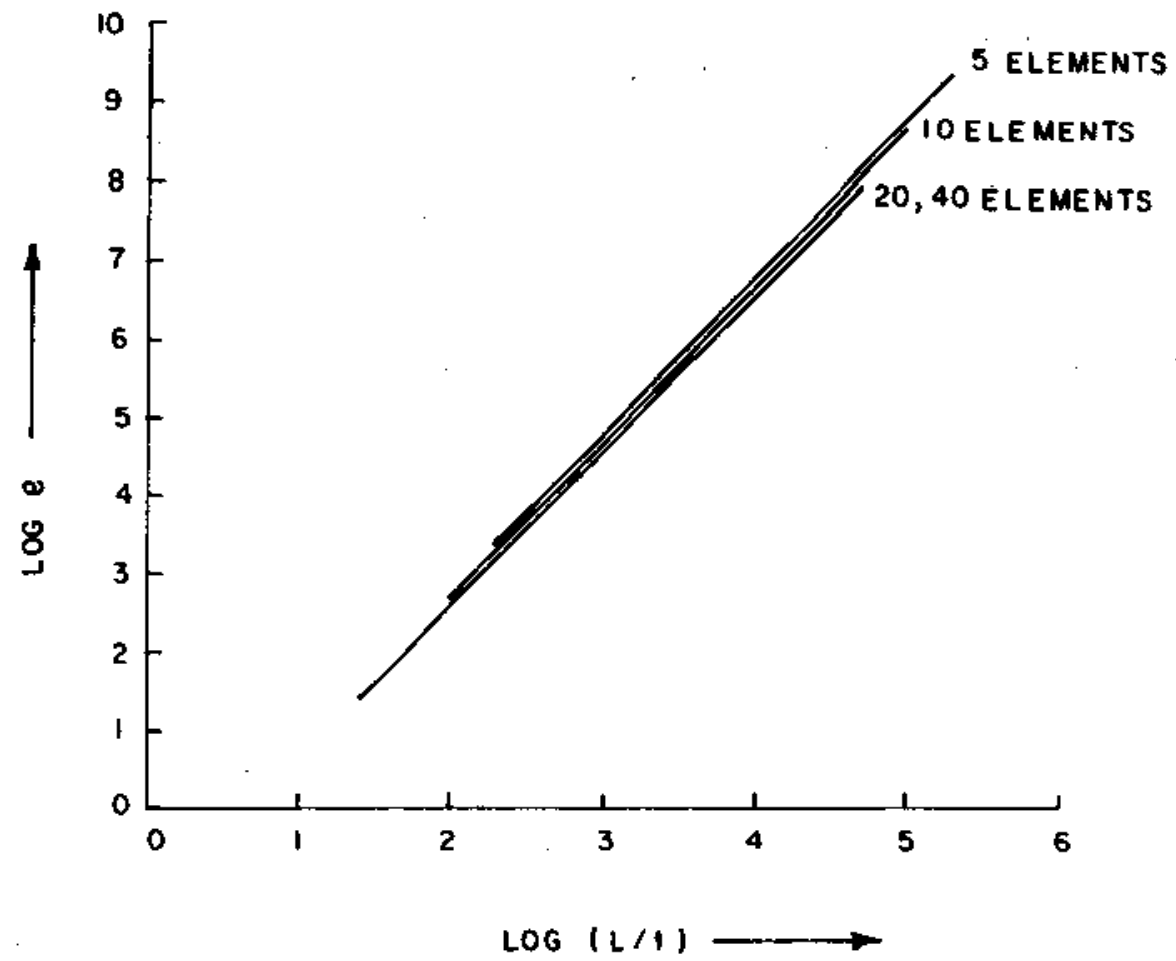

Figure 3. Additional stiffness parameter against $L / t$ for simply supported circular plate under UDL

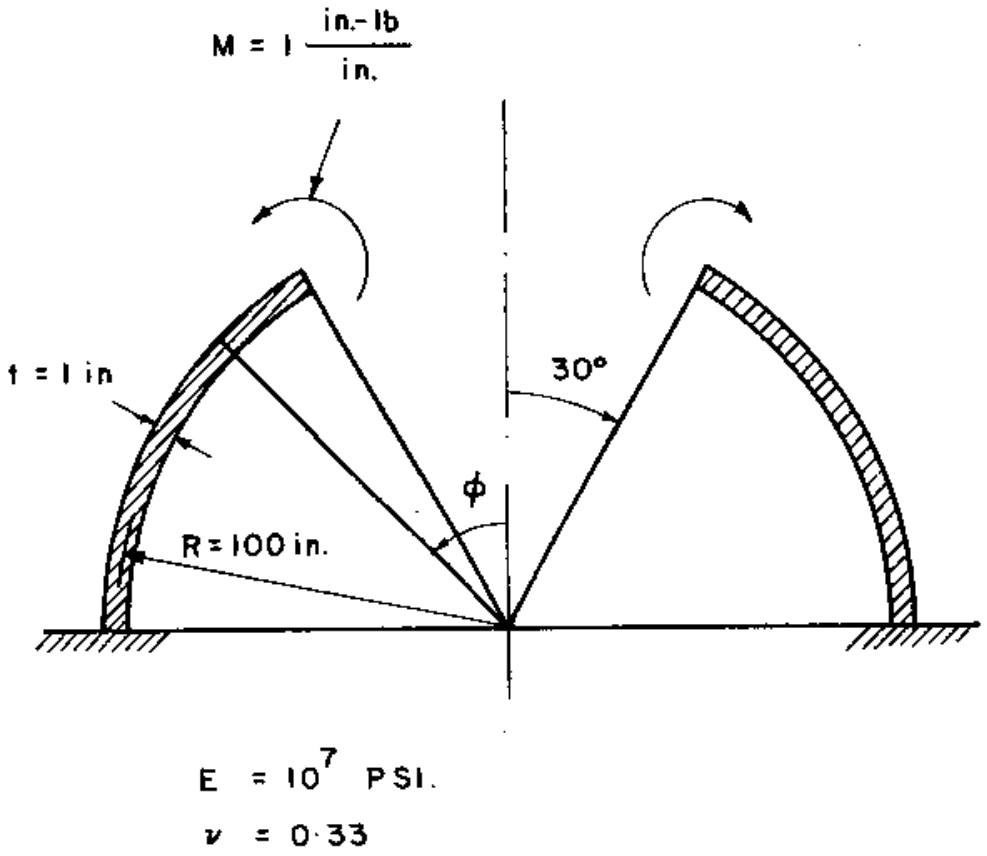

Figure 4. Clamped hemispherical shell under tip moment 
confirming that no errors of the second kind existed. It is clear now, why the Zienkiewicz element ${ }^{1}$ showed remarkable improvement with the reduced integration of the shear energy.

\section{Hemispherical shell under tip moment}

Figure 4 shows a hemispherical shell fixed fully along its base. Following Grafton and Strome, ${ }^{6}$ a tip moment $M$, of one in.lb/in. is applied to a shell of radius $R=100 \mathrm{in}$., $E=10^{7} \mathrm{psi}, v=0.33$ and $t=1 \mathrm{in}$. A modelling of the complete shell with $28 \mathrm{CMCS}$ elements $\left(14\right.$ at $0.5^{\circ}, 7$ at $1.0^{\circ}$, 3 at $2.0^{\circ}$ and 4 at $10^{\circ}$ intervals) produced a horizontal deflection at the tip of $1.595 \times 10^{-5}$ in. The tip moment, computed by extrapolating the bending stresses at the two Gauss integration points of the element at the tip, to the element nodes, gave a prediction of $0.993 \mathrm{in} . \mathrm{Jb} / \mathrm{in}$. - an accuracy of 0.7 per cent.

The 28-CMCS-element model was then used as the standard to compute the membrane and shear locking errors for a 6 element $\left(2\right.$ at $2^{\circ}, 1$ at $1^{\circ}, 1$ at $4^{\circ}, 1$ at $6^{\circ}, 1$ at $45^{\circ}$ intervals) and 10 element (6 at $1^{\circ}, 2$ at $2^{\circ}$ and 2 at $22.5^{\circ}$ intervals) configurations using the IMCS, CMCS, CMIS and IMIS models.

Figure 5 shows the horizontal displacement along the generator obtained by using a 10-element idealization with the various element types considered here. It is seen that the CMCS-10 modei predicts almost the exact solution. ${ }^{6}$ The errors obtained with the IMCS-10, CMIS-10 and IMIS-10

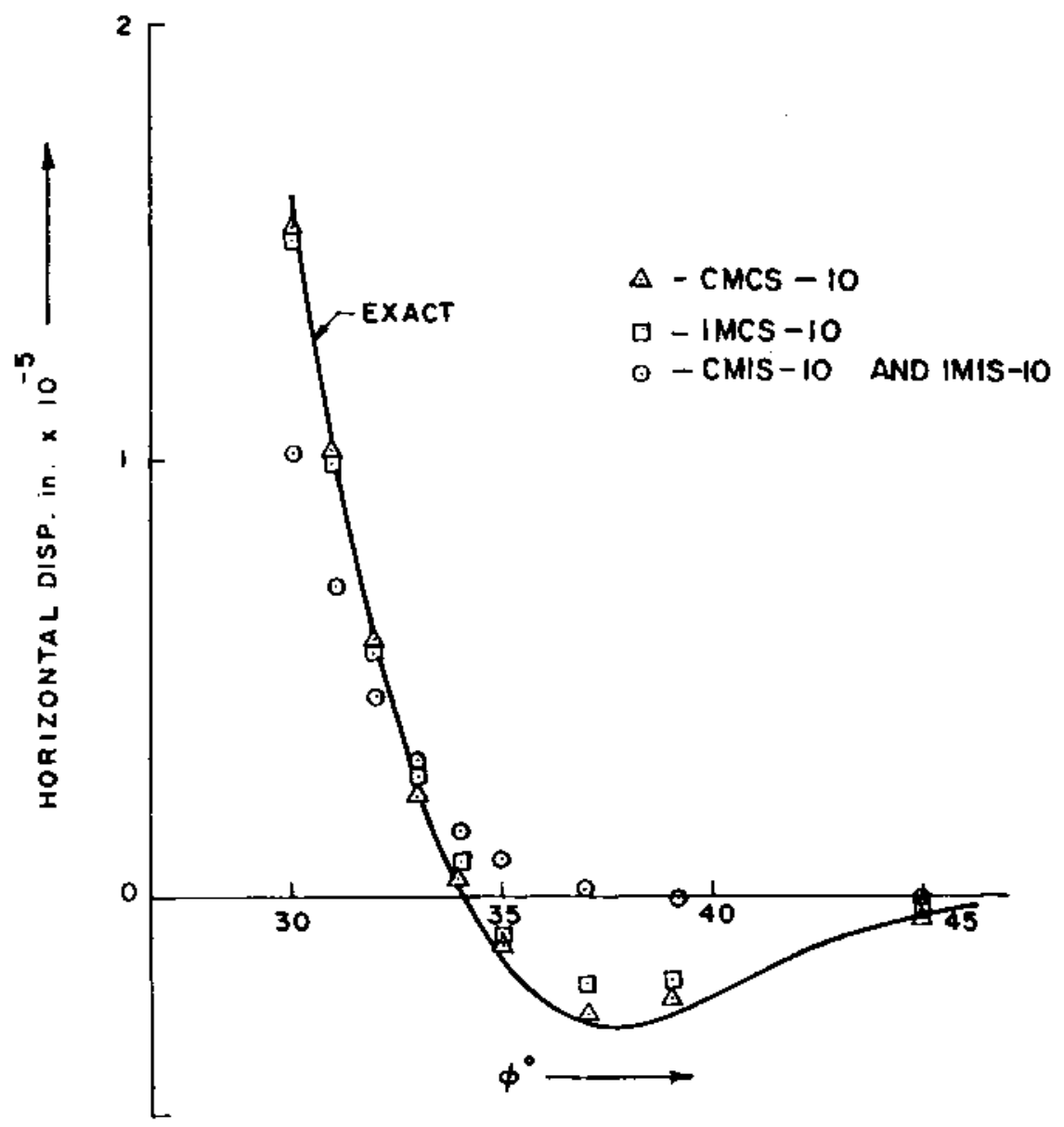

Figure 5. Hemispherical shell (see Figure 4) with one end clamped and a concentsated moment acting all along the other boundary. The plot shows horizontal displacement along the generator 


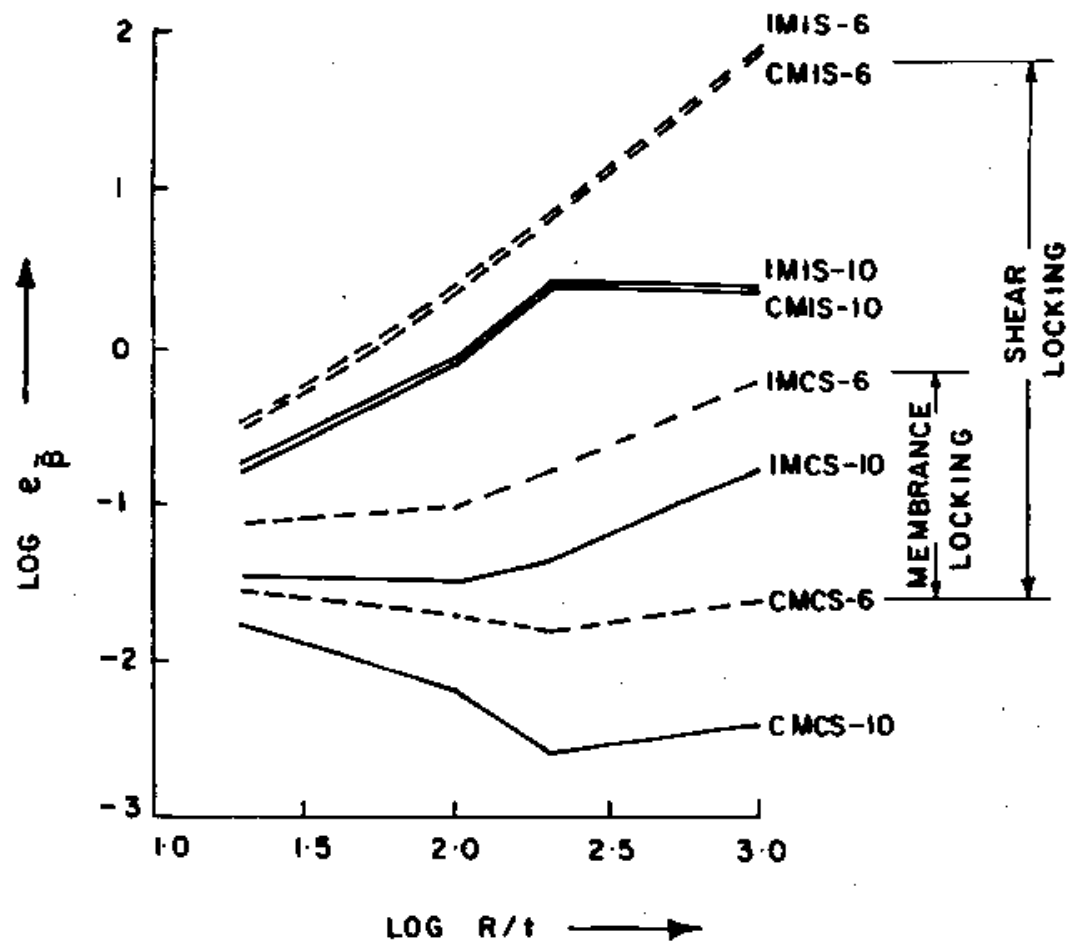

Figure 6. Additional stifness parameter $e_{\beta}$ against $R / t$ for a clamped hemispherical shell under tip moment

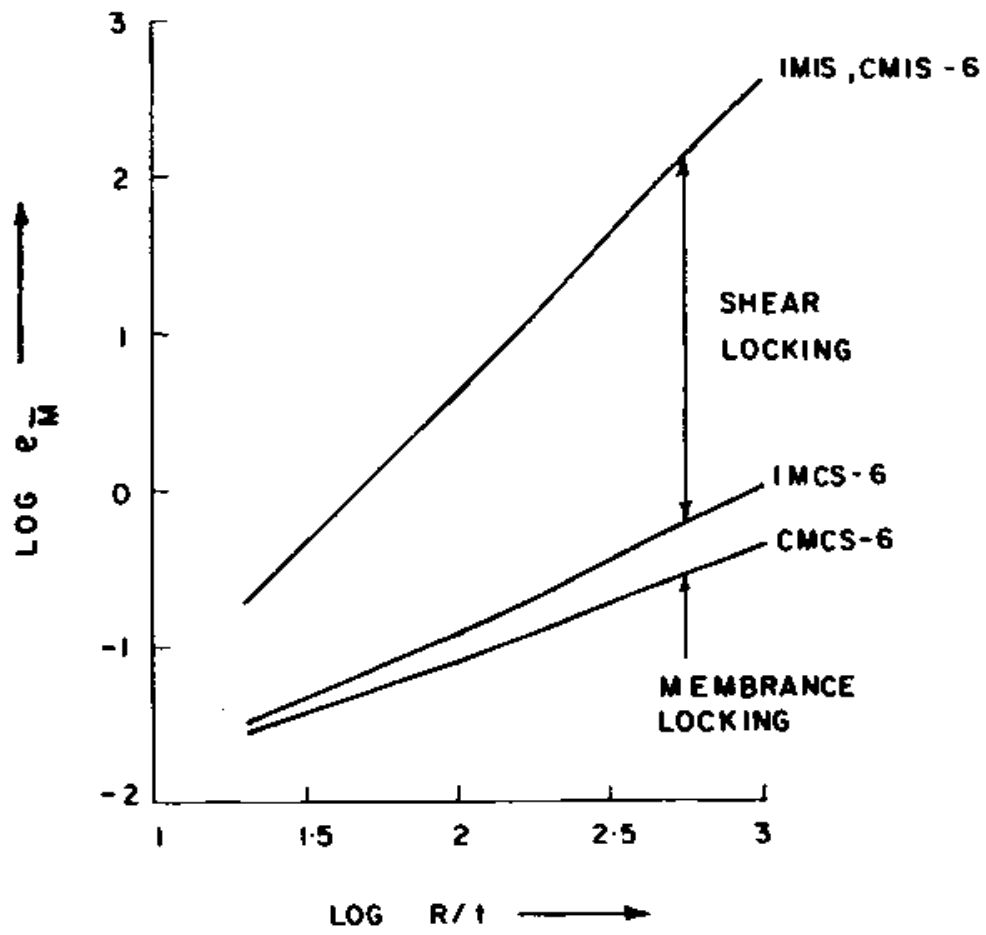

Figure 7. Additional stiffness parameter $e_{M}$ against $R / t$ for a clamped hemispherical shell under tip moment 


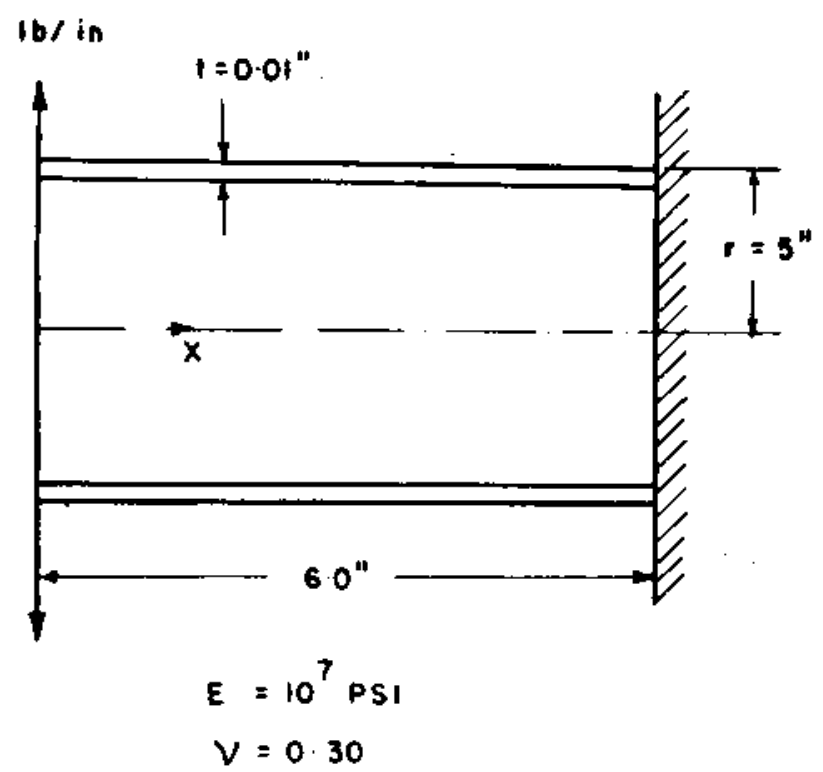

Figure 8. Cantilever cylindrical shell with ring load at the tip

models are noticeable. In order to assess the locking errors, the additional stiffness parameter error norm is used next.

In a typical case, the error norm ${ }^{5}$ is computed in terms of the section rotation $\bar{\beta}$ at the tip where the moment is applied. Thus,

$$
e_{\beta}(\mathrm{CMCS}-10)=\bar{\beta}(\mathrm{CMCS}-28) / \bar{\beta}(\mathrm{CMCS}-10)-1
$$

Figure 6 shows a plot on logarithmic scales of these errors as the shell thickness is reduced from $t=5 \mathrm{in}$. to $t=0.1 \mathrm{in}$. It is seen that shear locking errors predominate and that membrane locking effects are clearly perceptible, especially as the shell becomes thin. It is also seen that no simple rule for the variation of $e_{\beta}$ with $R / t$ exists, as the interactions of shear, membrane and bending energy in this doubly curved shell are quite complex and do not readily give a simple expression for the errors due to membrane or shear locking.

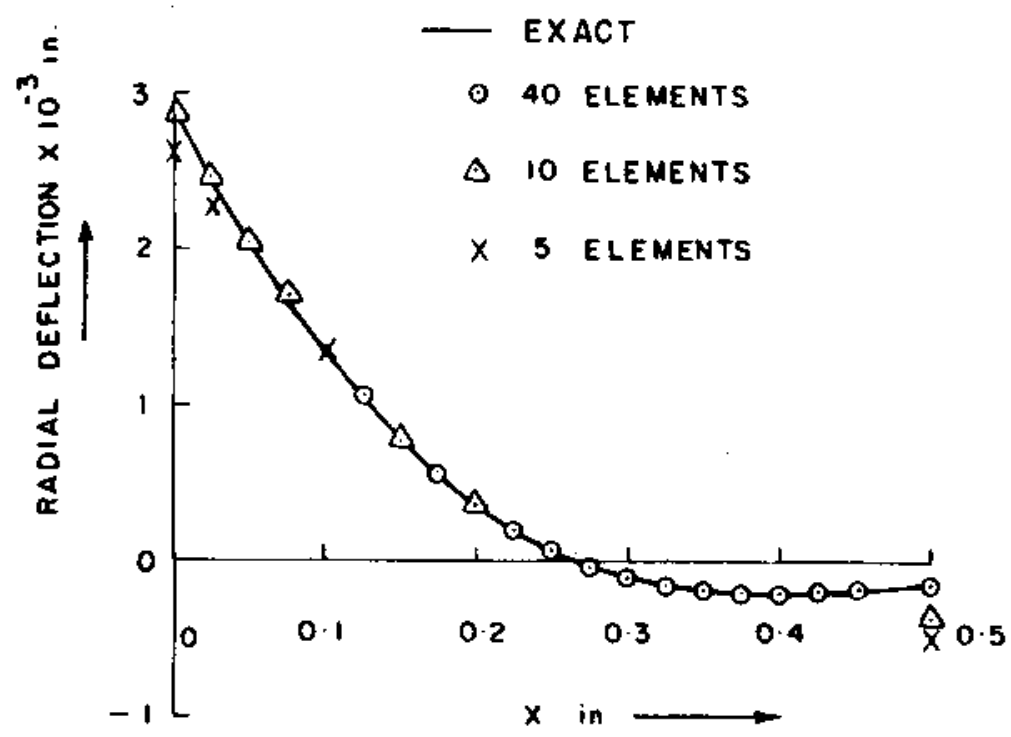

Figure 9. Radial displacements along cylindrical shell generator using CMCS model 
It is also instructive to consider an alternative form for the error norm, expressed this time in terms of the computed tip moment $\bar{M}$. Thus, in a typical case

$$
e_{M}(\mathrm{CMCS}-10)=\bar{M}(\mathrm{CMCS}-28) / \bar{M}(\mathrm{CMCS}-10)-1
$$

Figure 7 shows such a plot. Again, the shear locking and membrane locking etrors are quite clearly seen.

\section{Axisymmetrically loaded circular cylindrical shell}

Figure 8 shows a cantilever circular pipe acted upon by a uniformly distributed shear force of one $\mathrm{lb} / \mathrm{in}$. all along the free edge. The dimensions and elastic properties ${ }^{6}$ are as shown. Again, 5,10 and 40 element idealizations using all the element variations considered earlier are the basis for the finite element calculations.

Figure 9 shows the convergence trend for the radial deflections obtained by using the field consistent CMCS model. It is seen that a 10-element idealization produces very good accuracy. Figure 10 shows that similar accuracy is obtained with CMCS models for the distribution of meridional moment.

In order to study the errors due to shear and membrane locking, a shorter cylinder with $L=1.0 \mathrm{in}$., and the same radius and eiastic properties as above, is subjected to a uniformly distributed bending moment of one in.lb/in. along the free edge. The error norm is now defined by comparing the 5-element solution with the CMCS- 40 solution taken as the converged value.

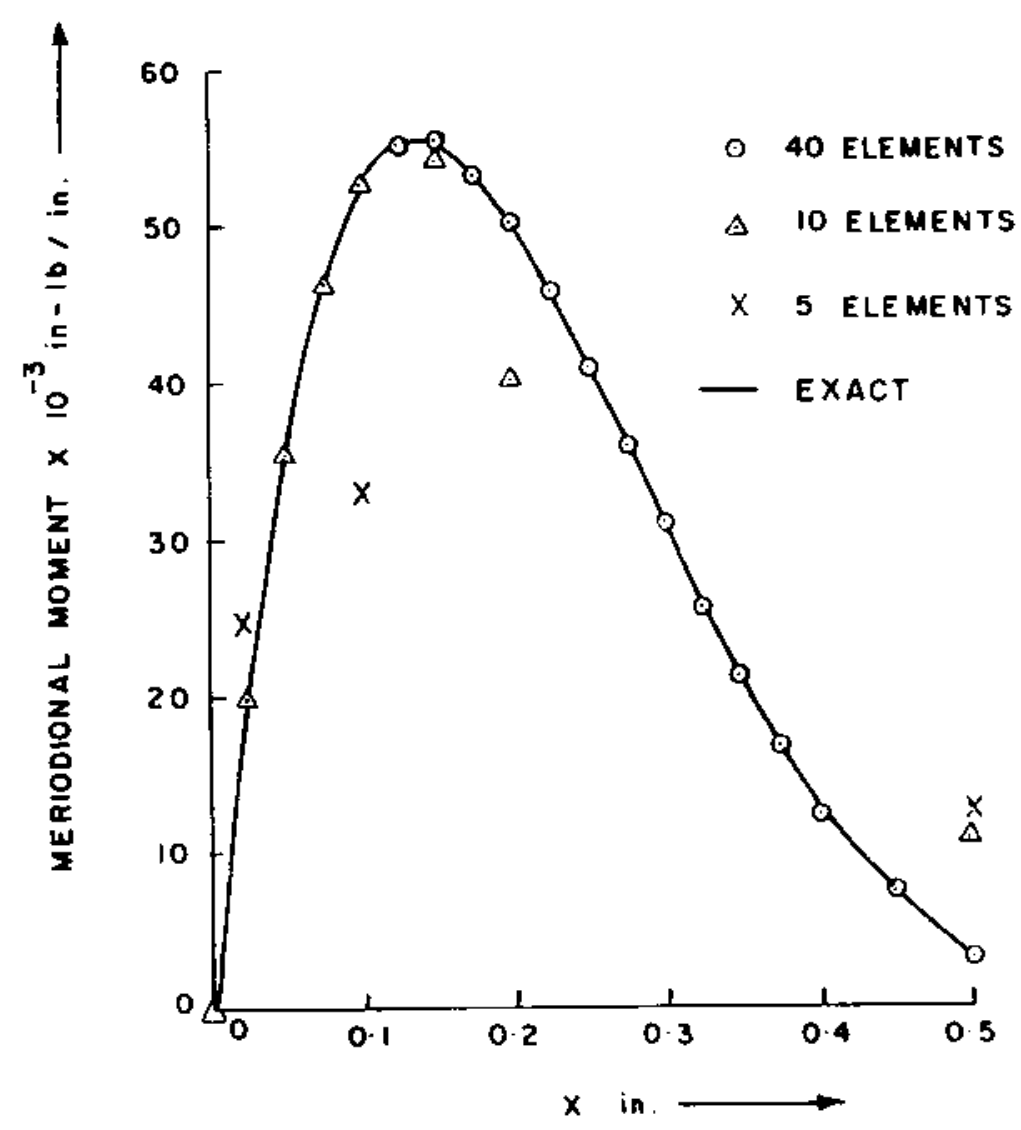

Figure 10. Meridjonal moment along cylindrical shell generator 


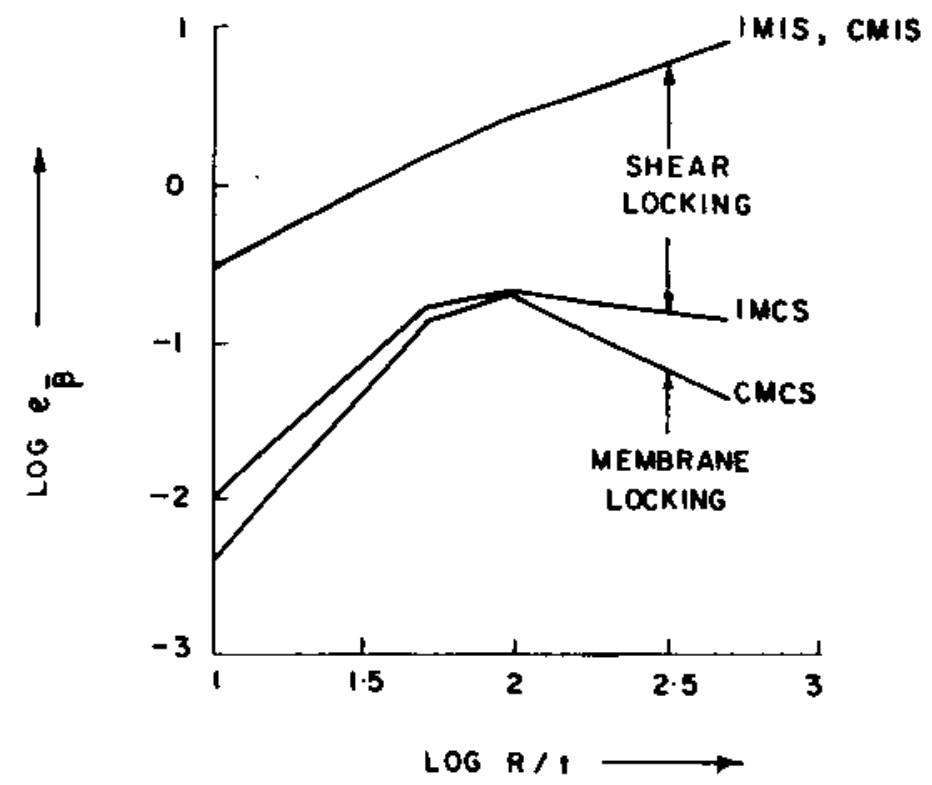

Figure 11. Additional stiffness parameter $e_{\beta}$ against $R / t$ for cantilever cylinder under tip ring loading

Thus, typically, where $\bar{\beta}$ is the section rotation at the free edge,

$$
e_{\bar{B}}=\bar{\beta}(\mathrm{CMCS}-40) / \bar{\beta}-1
$$

Figure 11 shows a plot on logarithmic scales of these errors as the shell thickness is reduced from $0.5 \mathrm{in}$. to $0.01 \mathrm{in}$. Shear locking errors are significant at low thicknesses. Membrane locking errors are perceptible but not very large.

Pressurized spherical shell

Figure 12 shows the details of a spherical shell subjected to uniform internal pressure. ${ }^{2}$ This

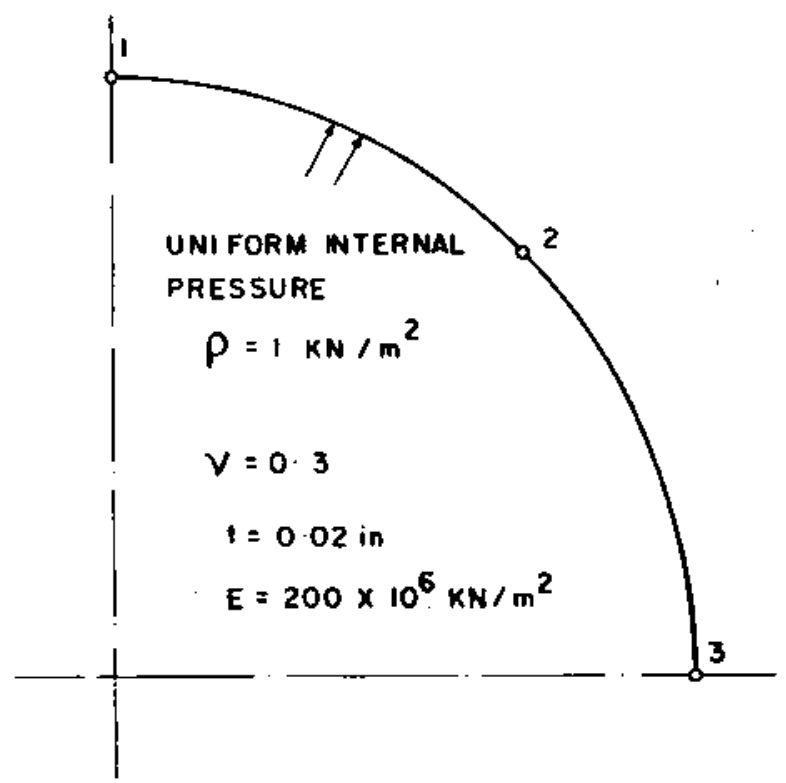

Figure 12. Sphere subjected to uniform internal pressure with two-linear-element idealization 
Table I. Convergence of radial displacements for two-CMCS-element idealization with increase in the order of integration (the order of integration is maintained the same for all energies)

\begin{tabular}{lccccccc}
\hline Integration order & 1 & \multicolumn{1}{c}{2} & 3 & 4 & 5 & \multicolumn{1}{c}{6} & Exact \\
\hline$w_{1} \times 10^{7} \mathrm{~m}$ & 0.753 & 0.815 & 0.829 & 0.837 & 0.842 & 0.845 & 0.875 \\
$w_{2} \times 10^{7} \mathrm{~m}$ & 0.854 & 0.884 & 0.886 & 0.888 & 0.889 & 0.889 & 0.875 \\
$w_{3} \times 10^{7} \mathrm{~m}$ & 0.956 & 0.953 & 0.944 & 0.939 & 0.935 & 0.933 & 0.875 \\
Error $^{*}$ & 21.9 & 9.8 & 7.0 & 5.7 & 4.9 & $\mathbf{4 . 5}$ & - \\
\hline
\end{tabular}

Error ${ }^{*}=\sum_{i=1}^{3}\left(w_{i}-w(\text { exact })\right)^{2} \times 10^{3}$

Table II. Effect of field-consistency-comparison of radial displacements for two CMCS-element and IMCS-element idealizations with a two-point integration rule

\begin{tabular}{lccc}
\hline Integration order & \multicolumn{2}{c}{2} & Exact \\
\hline Element type & CMCS & IMCS & - \\
$w_{1} \times 10^{7} \mathrm{~m}$ & 0.815 & 1.908 & 0.875 \\
$w_{2} \times 10^{7} \mathrm{~m}$ & 0.884 & 0.980 & 0.875 \\
$w_{3} \times 10^{7} \mathrm{~m}$ & 0.953 & 0.513 & 0.875 \\
\hline
\end{tabular}

Table III. Convergence in $w, N_{s}, N_{\theta}$ at $\theta=45^{\circ}$ with increase in the number of CMCS elements, using a four-point integration rule

\begin{tabular}{cccc}
\hline NEL & $w \times 10^{7} \mathrm{~m}$ & $N_{s}, \mathrm{kN} / \mathrm{m}$ & $N_{0}, \mathrm{kN} / \mathrm{m}$ \\
\hline 2 & 0.888 & 0.514 & 0.531 \\
3 & 0.879 & 0.505 & 0.513 \\
4 & 0.877 & 0.502 & 0.505 \\
5 & 0.8765 & 0.501 & 0.504 \\
6 & 0.876 & 0.500 & 0.503 \\
Exact & 0.875 & 0.500 & 0.500 \\
\hline
\end{tabular}

Table IV. Convergence in $M_{\theta}, N_{\theta}$, at nodes 1,2 and 3 with increase in the number of CMCS elements, using a four-point integration rule

Node 1

Node 2

Node 3

NEL

\begin{tabular}{lcccccc} 
& $M_{\theta}, \mathrm{kN}$ & $N_{\theta}, \mathrm{kN} / \mathrm{m}$ & \multicolumn{1}{c}{$\mathbf{M}_{\theta}, \mathrm{kN}$} & $N_{\theta}, \mathrm{kN} / \mathrm{m}$ & $M_{\theta}, \mathrm{kN}$ & $N_{\theta}, \mathrm{kN} / \mathrm{m}$ \\
\hline 2 & $-0.25 \times 10^{-5}$ & 0.521 & $0.14 \times 10^{-5}$ & 0.516 & $0.56 \times 10^{-6}$ & 0.517 \\
4 & $-0.22 \times 10^{-5}$ & 0.506 & $-0.19 \times 10^{-6}$ & 0.505 & $0.92 \times 10^{-7}$ & 0.506 \\
6 & $-0.32 \times 10^{-6}$ & 0.502 & $-0.33 \times 10^{-6}$ & 0.503 & $0.40 \times 10^{-7}$ & 0.503 \\
Exact & 0 & 0.500 & 0 & 0.500 & 0 & 0.500 \\
\hline
\end{tabular}


problem offers a critical test to evaluate the many special features which the present element accommodates. We shall consider first in idealization of half of the sphere by only two curved CMCS elements, each subtending a $45^{\circ}$ angle at the centre of the sphere. This modelling has only three nodes and the results obtained compare favourably with the eleven-node solution using quadratic curved elements of Mohr. ${ }^{2}$ Table I shows the radial deflection obtained at the three nodes (Figure 12) for the present two-element idealization as the order of integration is increased. The improvement obtained with increase in order of integration reflects the accuracy obtained by taking into account the improved geometrical description made possible by the curved element and also the need to integrate more accurately the $1 / r$ terms that occur in the definition of $\varepsilon_{\theta}$. The one-point integration will now reflect the errors that will be obtained with such coarse meshes when the Zienkiewicz' straight line element is used for such an example.

Another interesting feature to be considered is the specific problem requirement that the strains $\varepsilon_{s}$ and $\varepsilon_{\theta}$ are equal. For this equality to be achieved our field-consistency arguments recommend that $\varepsilon_{s}$ and $\varepsilon_{\theta}$ must be similarly interpolated. Table II now shows a comparison between the IMCS and CMCS models, but with a two-point integration rule for all energy terms. This demonstrates clearly the role field-consistent interpolations play in satisfying this specific problem need.

Table III shows the convergence obtained with an increase in the number of CMCS elements, using now a four-point integration rule for all energy terms, where $w, N$, and $N_{\theta}$ are radial displacement, and the meridional and circumferential force/unit thickness at $\phi=45^{\circ}$ of the shell. Table IV shows the convergence in $M_{\theta}$ and $N_{\theta}$ at nodes 1, 2 and 3 (Figure 12) obtained for the same set of examples. It is seen that $M_{\theta}$ is very nearly zero, and that $N_{\theta}$ varies little from pole to equator for the refined meshes.

\section{Pressurized torus}

Figure 13(a) shows the details of torus subjected to a constant internal pressure. Owing to the prevailing symmetry of the torus about its central horizontal plane, one half of the shell is idealized using 18 unevenly spaced CMCS elements, with more elements being crowded at the pole. The nodal points are placed at $180^{\circ}, 150^{\circ}, 120^{\circ}, 105^{\circ}, 100^{\circ}, 92^{\circ}, 91.5^{\circ}, 91 \cdot 0^{\circ}, 90 \cdot 5^{\circ}, 90 \cdot 0^{\circ}, 89 \cdot 5^{\circ}$, $89.0^{\circ}, 88.5^{\circ}, 88.0^{\circ}, 80.0^{\circ}, 75 \cdot 0^{\circ}, 60.0^{\circ}, 30 \cdot 0^{\circ}$ and $0.0^{\circ}$. The geometrical, material and loading details are given in the same Figure. The computed maximum radial displacement of $0.4272 \times 10^{-4} \mathrm{in}$. is observed at $\phi=100^{\circ}$. Since numerical values for comparison are not available, the computed radial displacements and membrane forces are shown in Figures 13(b)-(d). The very good agreement of the present results with those reported indicates the versatility of the present element.

\section{Branching shell ${ }^{6}$}

As the last exercise in this section a branching shell example is considered. Figure 14(a) shows a typical joint in a missile in which the shells are pressurized and also carry thrust loads. The isotropic material properties, loading and geometrical details are shown in the same Figure. The full shell is analysed using $38 \mathrm{CMCS}$ elements with finer mesh at the branching point. Ten CMCS elements are used to model the hemispherical portion with nodes placed at $90.0^{\circ}, 60 \cdot 0^{\circ}, 33 \cdot 0^{\circ}$, $18.0^{\circ}, 7.5^{\circ}, 5.0^{\circ}, 3.0^{\circ}, 1.5^{\circ}, 0.5^{\circ}$ and $0.0^{\circ}$. The segments $\mathrm{AC}$ and $\mathrm{CF}$ are divided into 14 elements each of lengths in inches $1-5,1-3,3$ at 1, 0-3, 0-2 and 7 at $0-1$. As far as boundary conditions are concerned the point $A$ is clamped and symmetry conditions are applied at the point $D$. The computed maximum radial displacement of 0.127 in. is observed at the point $F$. 


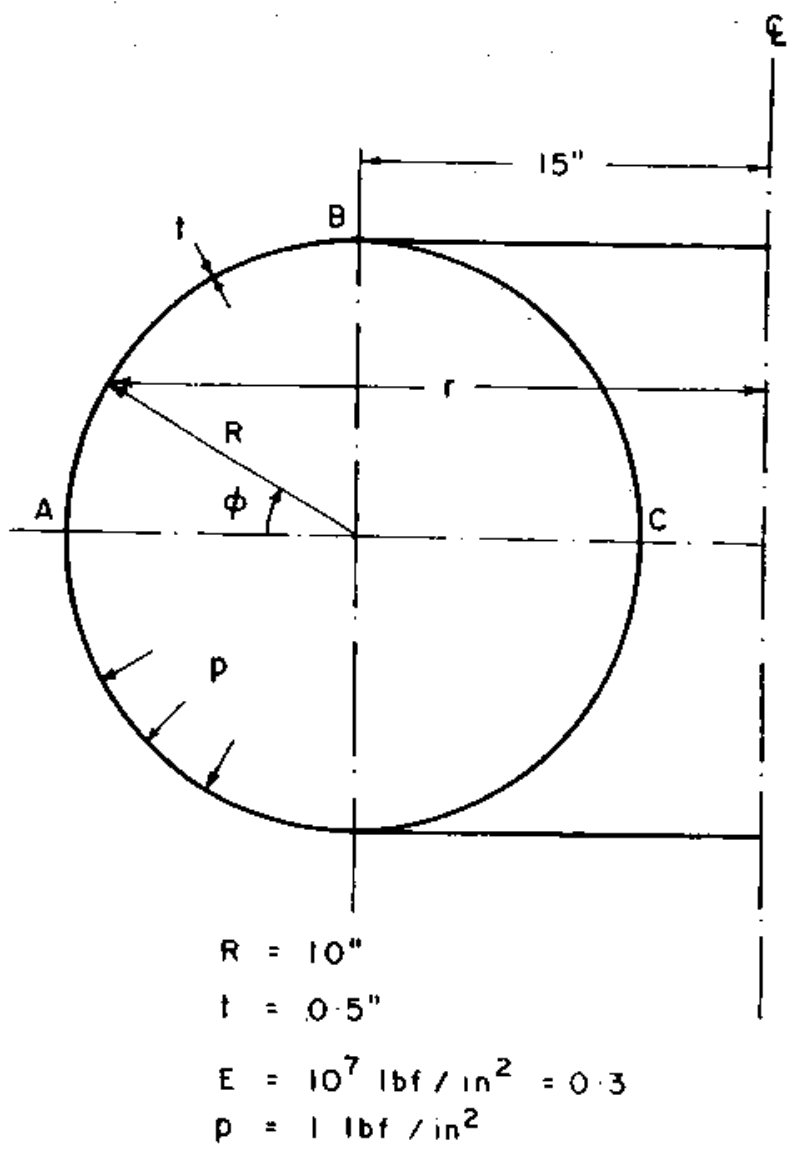

Figure 13(a). Torus shell model

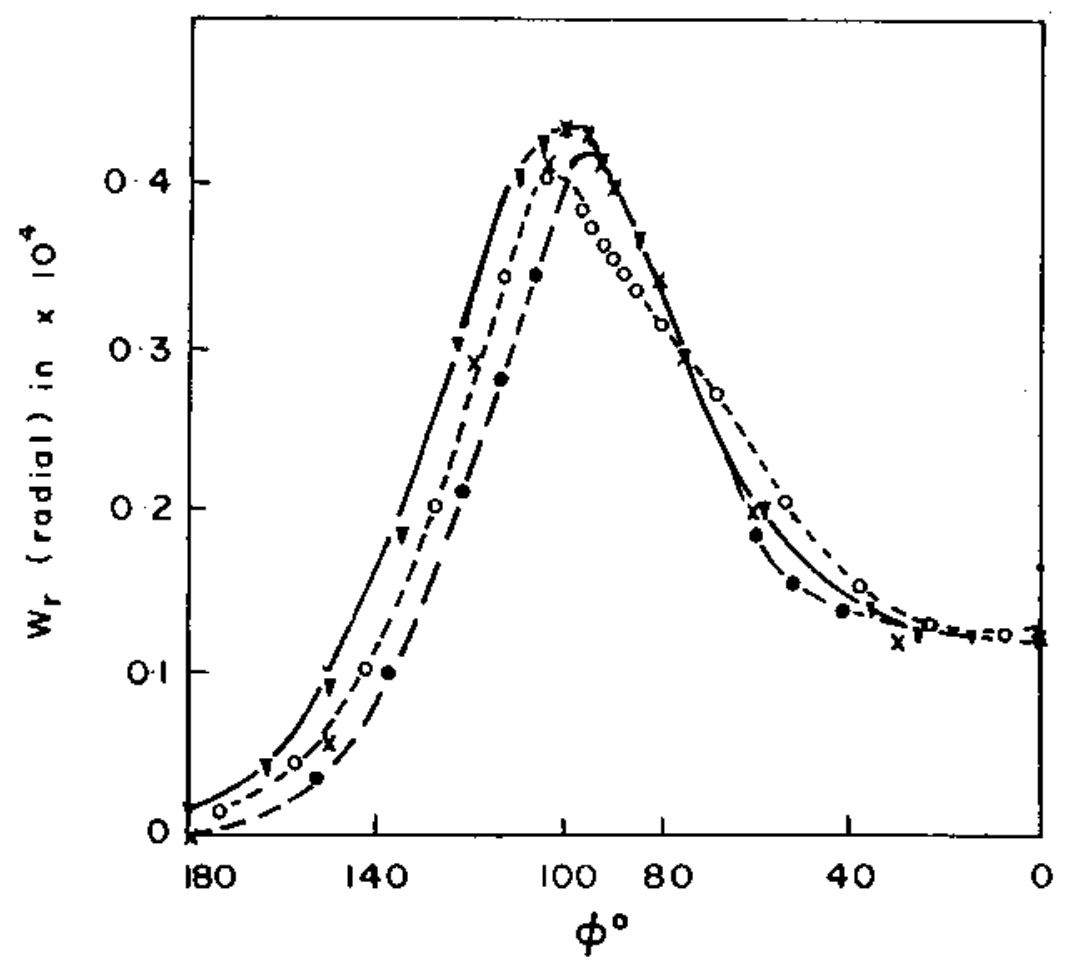

Figure 13(b). Toroidal shell under internal pressure: Chan and Firman (9),- - Giannini and Miles (8),- $\mathrm{O}-$ Delpak (7); $\nabla$ Zienkiewicz et al. (1); $\times$ Present CMCS 


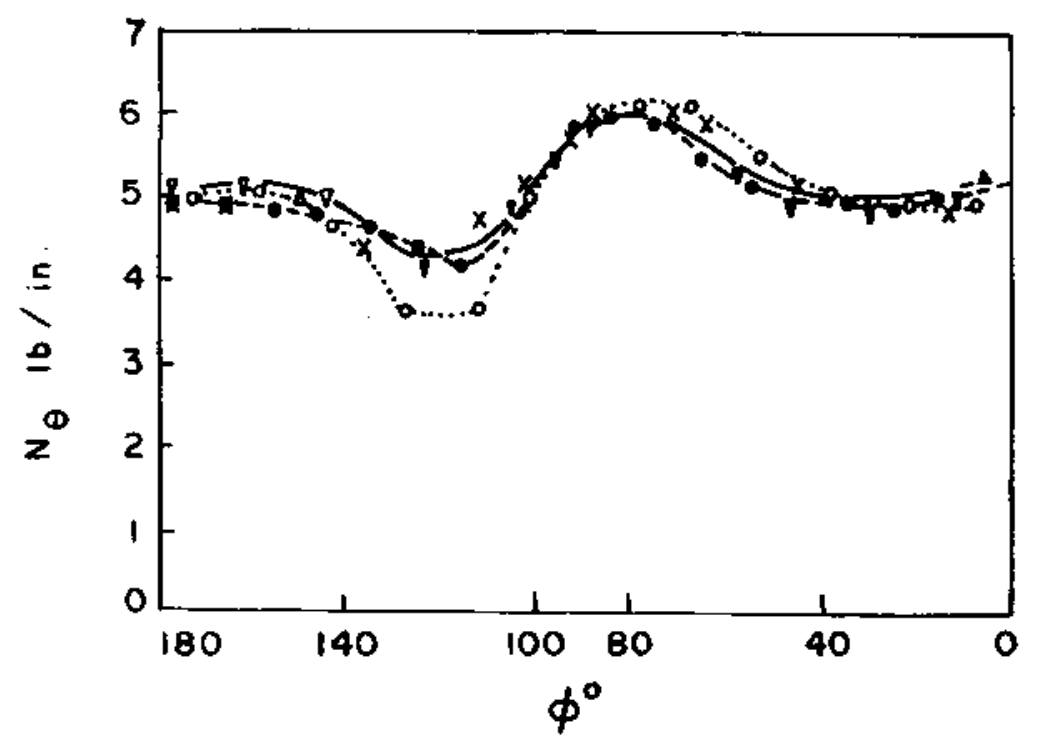

Figure 13(c). Distribution of $N_{0}$ on the segment ABC

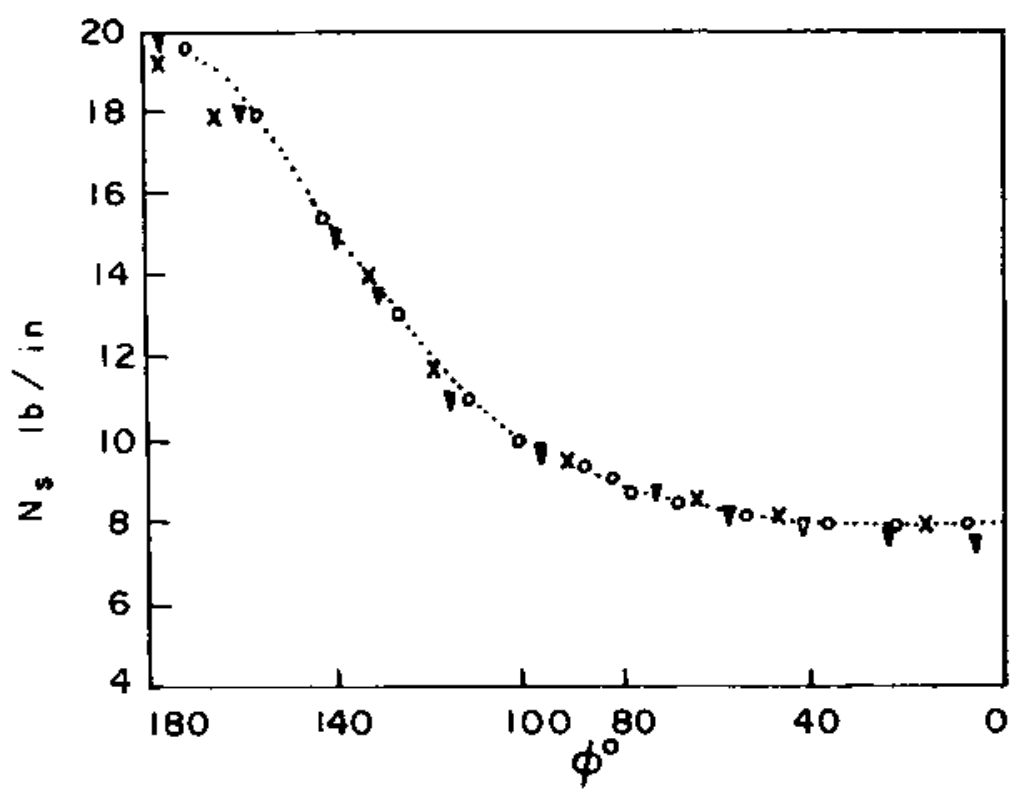

Figure 13(d). Distribution of $N_{s}$ on the segment ABC:

Chan and Firman (9);

-... Giannini and Miles (8);

- O-Delpak (7):

7 Zienkiewicz et al. (1);

$\triangle$ Jordan (11)

$\nabla \quad$ Sanders and Liepins $(10)$

$x$ Present CMCS 


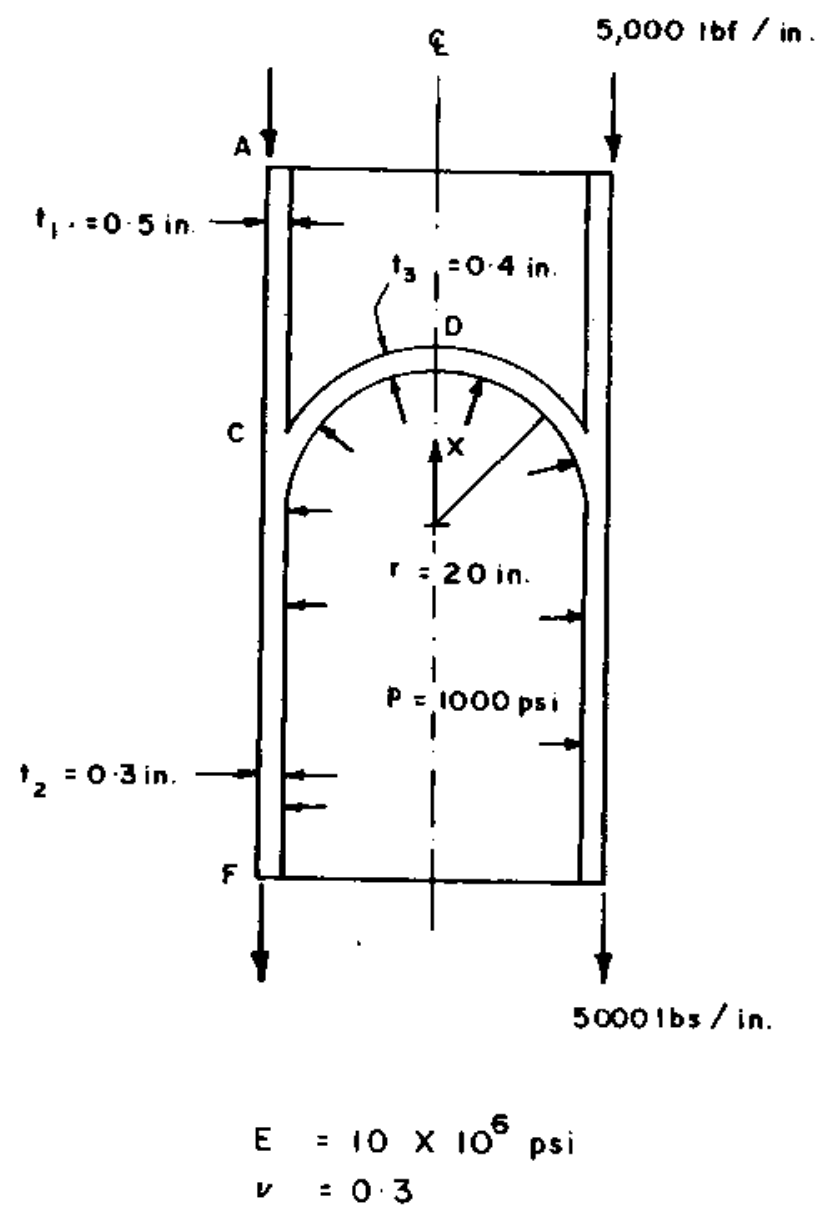

Figure 14(a). Head cylinder skirt junction

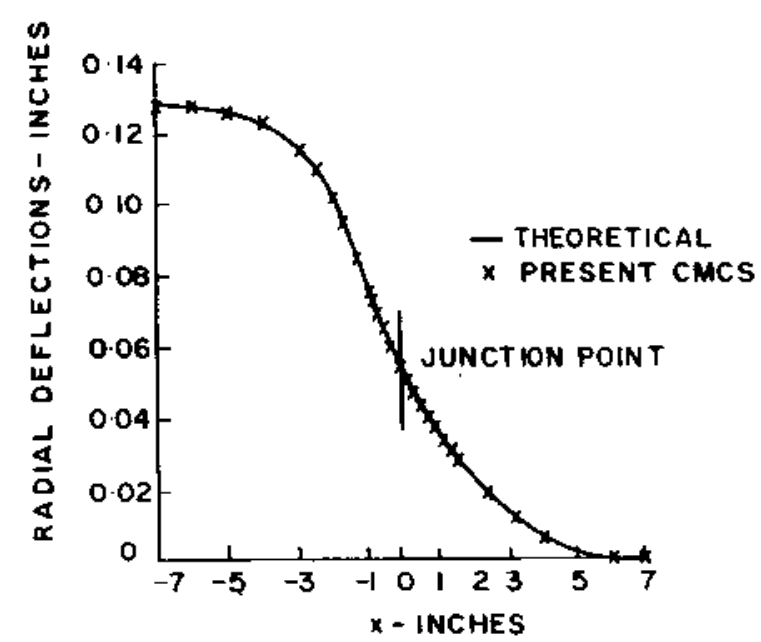

Figure 14(b). Radial deflections along direction of cylinder axis near junction point 
As no numerical values are available for comparison, the plot of radial displacements along the generator ACF is shown in Figure 14(b). It is clear from the Figure that the theoretical distribution of radial displacement is identical to the CMCS nodal output.

\section{CONCLUSIONS}

The field-consistency interpretation allows a simple two-noded curved axisymmetric shetl element to be designed free of shear and membrane locking errors and with greater flexibility as to design for specific problem needs. Its efficiency in predicting deflections and stresses has been demonstrated through several examples.

\section{ACKNOWLEDGEMENTS}

The authors are deeply indebted to Dr. B. R. Somashekar, Head, Structures Division for his constant encouragement and support.

\section{REFERENCES}

1. O.C. Zienkiewicz, J. Bauer, K. Morgan and E. Onate. 'A simple and efficient element for axisymmetric shells', Int. $j$. numer. methods eng., 11, 1545-1558 (1977).

2. G. A. Mohr, 'Applications of penalty functions to a curved isoparametric axisymmetric thick shell element', Comp. Struct., 15, 685-690 (1982).

3. G. Prathap and G. R. Bhashyam, 'Reduced integration and the shear flexible beam element', Int.j. numer. methods eng., 18, 195-210(1982).

4. G. Prathap, 'The curved beam/deep arch/finite ring element re-visited', Int. j. numer. methods eng., 21, 389-407 (1985).

5. G. Prathap, 'An additional stiflening parameter measure of error of the second kind', Int. j. numer. methods eng., 21, $1001-1012$ (1985).

6. P. E. Grafton and D. R. Strome, 'Analysis of axisymmetric shells by the direct stiffness method', A.I.A.A. J., 1, 23422347 (1963).

7. R. Delpak, 'Role of the curved parametric element in linear analysis of thin rotational shells', Ph.D. Thesis, Department of Civil Engineering and Building, The Polytechnic of Wales, 1975.

8. M. Giannini and G. A. Miles, 'A curved element approximation in the analysis of axisymmetric thin shells', Int. $j$. numer. methods eng., 2, 459-476 (1970).

9. S. Ahmad, B. M. Irons and O. C. Zienkiewicz, 'Curved thick shell and membrane elements with particular reference to axi-symmetric problems', Proc. 2nd Conf. Matrix Methods Struct. Mech., Wright-Patterson A. F. B., Ohio, AFFDLTR-68-150 (1968).

10. J. L. Sanders Jr. and A. Liepins, 'Toroidal membrane under internal pressure', A.I.A.A. J., I, 2105-2110 (1963).

11. F. F. Jordan, 'Stress and deformations of the thin-walled pressurised torus', J. Aerospace $S c$, 29, 213-225 (1962). 